\title{
La Educación Ambiental en el S. XXI (página en construcción, disculpen las molestias)
}

\author{
María-José Bautista-Cerro \\ Facultad de Educación. UNED. Cátedra UNESCO de Educación Ambiental y Desarrollo \\ Sostenible. c/ Juan del Rosal, 14. 28040 Madrid. España. \\ mjbautistac@edu.uned.es \\ ORCID: https://orcid.org/0000-0002-3658-2191 \\ Ma Ángeles Murga-Menoyo \\ Facultad de Educación. UNED. Cátedra UNESCO de Educación Ambiental y Desarrollo \\ Sostenible. c/ Juan del Rosal, 14. 28040 Madrid. España. \\ mmurga@edu.uned.es \\ ORCID: http://orcid.org/0000-0001-8779-6192 \\ María Novo \\ Catedrática Emérita de la UNED. Titular de la Cátedra UNESCO de Educación Ambiental \\ y Desarrollo Sostenible. c/Juan del Rosal, 1428040 Madrid. España. \\ mnovo@ecoarte.org \\ ORCID: http://orcid.org/0000-0002-6822-5658
}

[Recibido: 1 Febrero 2019. Revisado: 8 Febrero 2019. Aceptado: 12 marzo 2019]

\begin{abstract}
Resumen: La educación ambiental es un movimiento con un largo e intenso recorrido histórico y, en este artículo, realizamos un breve repaso por algunos de los hitos más importantes y su situación actual. Los problemas ambientales, principalmente el cambio climático han legitimado las tesis que desde la educación ambiental se vienen difundiendo desde hace décadas: la necesidad de un cambio en las relaciones del ser humano con el medio natural y con sus congéneres. Sin embargo, los problemas externos e internos que ha experimentado han dificultado su avance y consolidación. Más allá del necesario examen crítico no podemos dejar de celebrar no solo la pervivencia sino la vitalidad de un movimiento más que necesario, junto con otros, en el camino a la sostenibilidad.
\end{abstract}

Palabras clave: Educación Ambiental; Educación para la Sostenibilidad, crisis ambiental, desarrollo sostenible.

Environmental education in the 21st century (page under construction, sorry for the inconvenience)

Abstract: Environmental education is a movement with a long and intense historical journey and we briefly review of some of the most important milestones and their current situation. Environmental problems, mainly climate change, have legitimized the theses that have been spreading from environmental education for decades: the need for a change in the relations of the human being with the environment and with its congeners. However, the external and internal problems that it has experienced have hindered its progress and consolidation. Beyond the necessary critical examination we need to celebrate not only the survival but the vitality of a movement more than necessary, along with others, on the road to sustainability.

Keywords: Environmental education, education for sustainable development, environmental crisis, sustainability.

Para citar este artículo: Bautista-Cerro, M. J., Murga-Menoyo, M. A. y Novo, M. (2019). La Educación Ambiental (página en construcción, disculpen las molestias). Revista de Educación Ambiental y Sostenibilidad 1(1), 1103. doi: 10.25267/Rev_educ_ambient_sostenibilidad.2019.v1.i1.1103 


\section{Del éxito como especie al fracaso ambiental}

Entre la cantidad de información que recibimos sobre la situación actual del planeta, bien a través de los diarios, Internet, la abundante bibliografía especializada, o bien la propia experiencia personal, podemos encontrar suficientes noticias que nos llevan a pensar que nos encontramos atravesando una crisis ambiental sin precedentes. Nunca antes tuvimos acceso a tantos datos, a tanta información, nunca antes habíamos asistido de manera alterna y continuada a escenas de ficción y realidad con la capacidad de producir el mismo miedo, el mismo malestar, la misma indignación. Pero, como ya anunciaba Sartori (1998), la primacía de lo visible lleva al homo sapiens a convertirse en homo videns, en una dinámica que ha acabado con el pensamiento abstracto $\mathrm{y}$, por tanto, con la capacidad reflexiva y critica del ser humano. La profusión de historias y problemáticas nos lleva a tomar distancia frente a una situación absolutamente abrumadora y cuyas posibles soluciones están en disonancia con los modelos de vida y consumo con los que hemos crecido y se presentan como legítimos y deseables.

Parece que hemos dejado atrás las teorías negacionistas ante las evidencias que, año a año, aportan, entre otros, los informes del Panel Intergubernamental de Cambio Climático (IPCC) sobre lo alarmante de nuestra situación y sobre la necesidad de tomar medidas concretas y urgentes para mejorarla. Sin embargo, ni los gobiernos ni las empresas, en general, son capaces de diseñar y ejecutar acciones contundentes que faciliten procesos, no tanto de solución al cambio climático, cuyos efectos ya son irreversibles en muchos aspectos, sino, ni siquiera, de la mitigación y adaptación que serían efectivas.

Ciertamente son cada vez más numerosos los movimientos y las voces que claman por un cambio de rumbo y que diseñan caminos alternativos al actual modelo energético, agroalimentario, educativo, etc. Las críticas no son nuevas; con los procesos de industrialización, el capitalismo y, con ello, el auge de la idea utilitarista de la naturaleza, aparecen los movimientos ambientalistas y ecologistas, entre cuyos líderes pioneros Thoreau ocupa un lugar relevante. Desde entonces, mucho se ha argumentado sobre la necesidad de afrontar los peligros de nuestros modelos de producción, distribución y consumo (Carson, 1962; Meadows, 1972, entre otros). El agravamiento de la situación ha evidenciado que no solo estamos ante un mero deterioro ambiental, hemos ido más allá. Duarte propone la idea de cambio global como el conjunto de cambios ambientales afectados por la actividad humana, con especial referencia a cambios en los procesos que determinan el funcionamiento del sistema Tierra (2006, p. 23). Y la teoría del Antropoceno, que ha obtenido una amplia difusión -impulsada por el Nobel de Química, Paul Crutzen-, viene a afirmar que el planeta Tierra ha entrado en un nuevo período geológico debido a la acción del ser humano.

Señala Harari (2015) que ha habido tres revoluciones importantes que han incidido en el mundo tal y como lo conocemos: la revolución cognitiva, hace 70.000 años; la revolución agrícola, hace unos 12.000 años; y, más recientemente, la científica hace solo 500 años. Si bien podemos afirmar nuestro triunfo como especie, al haber sido capaz de colonizar todos los hábitats y adaptarnos a las diversas condiciones climáticas y meteorológicas, lo cierto es que ese camino ha supuesto que el planeta haya alcanzado un punto crítico en el que los sistemas que sustentan la vida se 
encuentran seriamente dañados y su futuro comprometido. Podemos hablar, por tanto, de que nuestro éxito como especie nos aboca, si no cambiamos el rumbo, a un fracaso ambiental sin precedentes en la acción de cualquier otra especie.

Destacados científicos, Vitousek, Mooney, Lubchenco y Melillo (1997) han propuesto hace décadas un modelo para considerar los efectos de la actividad humana sobre el planeta. Para ello, toman como referencia el crecimiento de la población y el aumento en el uso de recursos, estableciendo así una relación directa entre estas magnitudes y el impacto en la biosfera. Para estos autores, las actividades humanas han provocado, entre otras, dos consecuencias graves a escala planetaria: el cambio climático y la pérdida de diversidad biológica.

Cabe pues pensar que el momento actual y la crisis ambiental se caracterizan por un mundo complejo y multicéntrico, fuertemente descompensado, en el que interactúan actores diversos en un escenario definido por los conflictos entre actores e intereses donde además los problemas son sinérgicos (Novo, 2006, p. 56).

En la situación actual se hace preciso atraer la atención sobre las causas y procesos que nos han conducido hasta ella. Sin duda, hay unos valores dominantes que sustentan y legitiman el modelo, y que son fruto de la evolución del pensamiento occidental, principalmente del pensamiento económico. La humanidad se encuentra bajo un desarme político provocado por grandes grupos económicos que no quieren cambiar sus modelos de negocio mientras sigan obteniendo amplios beneficios. La democracia misma se ve comprometida, así como los medios para hacer frente a la lógica económica dominante, sostener los espacios de vida no mercantiles, mantener instituciones dependientes de principios ajenos al provecho, corregir o atenuar los efectos de la ley de la competencia mundial (Laval y Dardot, 2015, p. 18).

Entender el paradigma que nos ha traído a esta situación es el primer paso; el segundo es su cuestionamiento. El cambio de paradigma no sucede de manera casual ni inmediata y, en este caso, la educación tiene un papel crucial. Tal y como afirma Gimeno Sacristán (2001), la educación tiene una doble vertiente: una propositiva y otra crítica. La propositiva se centra en el desarrollo de una idea compartida de comunidad; y la segunda, la crítica, en la formación de ciudadanos y ciudadanas que sean capaces de re-pensar la comunidad para mejorarla, cada uno desde su posición.

\section{Evolución histórica de la Educación Ambiental}

Comúnmente se sitúan los comienzos de la educación ambiental (EA) en torno a los años 70. La Unesco hizo manifiesto su interés en la materia y presentó, en 1968, el Estudio comparativo sobre el medio ambiente en la escuela. En él quedaba plasmada la idea de que la EA no debe concretarse en una nueva disciplina, sino en una línea de trabajo transversal a lo largo del currículo escolar. Igualmente, se apostaba por la importancia de considerar el ámbito más cercano como el punto de partida para el estudio del medio ambiente.

Uno de los eventos más importantes de la década y para el desarrollo posterior de la EA tuvo lugar en Estocolmo, en 1972. La Conferencia de las Naciones Unidas sobre Medio Ambiente Humano consiguió la participación de un elevado número de países. Asistieron 113 miembros de las Naciones Unidas, en un encuentro marcado por el contexto político de la época, la Guerra Fría. Además recordemos que en los países 
desarrollados estaba cobrando fuerza la corriente ambientalista, que tomaba posiciones en contra de las consecuencias del modelo industrial y que estaba presente en obras como Primavera silenciosa (Carson, 1962), La tragedia de los comunes (Hardin, 1968), o Los límites del crecimiento (Meadows, Meadows y Randers, 1972). De la conferencia se obtuvieron dos documentos principales: La Declaración sobre Medio Ambiente Humano y el Plan de Acción para el Medio Ambiente Humano, siendo uno de los logros de esta Conferencia introducir la temática ambiental como una línea de trabajo de los gobiernos, lo que se concreta en el crecimiento exponencial de Ministerios de Medio Ambiente en la década de los 70. Además, surgieron dos programas de gran importancia: el Programa de las Naciones Unidas para el Medio Ambiente (PNUMA) y el Programa Internacional de Educación Ambiental (PIEA).

El PNUMA incide en la importancia de la EA, dirigida a un público extenso, y de la formación y capacitación ambiental, orientada a profesionales de diversos ámbitos y responsabilidades.

Por su parte, el PIEA establecía, entre sus objetivos promover tanto la elaboración y evaluación de nuevos materiales, planes de estudio, materiales didácticos y programas, en el campo de la EA como el adiestramiento y actualización de personal clave para su desarrollo.

En el marco del PIEA, y como su plataforma de lanzamiento, se celebró, en 1975, el Seminario Internacional de Educación Ambiental, en la ciudad de Belgrado. En él se firmó la Carta de Belgrado, en la que se cuestiona el concepto de desarrollo vigente en la mayor parte del mundo económico y se insta a un replanteamiento del mismo. Recoge la Declaración de las Naciones Unidas sobre el Nuevo Orden Económico Internacional y asume su concepto de desarrollo como un proceso centrado en las necesidades de todos los ciudadanos de la Tierra, el equilibrio y la armonía de la humanidad y el medio ambiente.

Esta Carta sigue siendo un referente para la realización de cualquier programa de EA. Entre sus metas encontramos:

Lograr que la población tenga conciencia del medio ambiente y se interese por él y por sus problemas conexos y que cuente con los conocimientos, aptitudes, motivaciones $y$ deseos necesarios para trabajar individual $y$ colectivamente en la búsqueda de soluciones a los problemas actuales y para prevenir los que pudieran aparecer en lo sucesivo. (Unesco, 1978, p. 3).

Todo esto nos lleva a una EA centrada en la acción, que busca la participación y la movilización de los educandos. En este sentido es una educación que toma muy en cuenta el rol de quienes aprenden. No sólo se plantea hacer llegar unos conocimientos a los estudiantes sino que, entre sus objetivos, también se incluye la formación de actitudes y aptitudes, estimulando la participación activa de todos los miembros de la comunidad de aprendizaje.

Aparece de manera temprana, como hemos visto, la necesidad de formación de personal especializado (PNUMA) y de personas clave (PIEA). Este objetivo se verá reforzado en las Recomendaciones que se redactaron en el Seminario de Belgrado. 
Un año antes, en 1974, se había celebrado en Cocoyoc (México) un simposio de expertos sobre desarrollo y medio ambiente organizado por el PNUMA y la UNCTAD. El documento final, la Declaración de Cocoyoc muestra una desconfianza profunda en los mecanismos del mercado como solución a los problemas ambientales y de desarrollo (...) El nuevo paradigma del ecodesarrollo aparecía en la escena de la política mundial como alternativa a la problemática medioambiental y a la del desarrollo (Gutiérrez Bastida, 2011, p. 71). La Declaración de Cocoyoc no solo planteaba el problema del sub-desarrollo sino también del sobre-desarrollo, lo que significaba un fuerte cuestionamiento del orden dominante, un paradigma contrario a las corrientes neoliberales imperantes en la época y fue desterrado de las cumbres internacionales. Puede que los gobiernos estuvieran dispuestos a hablar de pobreza y subdesarrollo, de ayuda internacional e, incluso, de responsabilidad; pero no a poner en entredicho la necesidad del crecimiento y del mercado como motores de progreso y bienestar. Cuestión que aún en nuestros días continúa siendo motivo de controversia.

Dos años más tarde, en 1977, se llevó a cabo la Conferencia Intergubernamental de Educación Ambiental, en Tbilisi (Georgia, U.R.S.S). Es considerada como el acontecimiento más significativo de la historia de la EA pues en ella se aprobaron los pilares de su corpus teórico. Se trata de referentes anticipatorios de muchas de las ideas que aún hoy se consideran innovadoras en ciertos ámbitos, renuentes a aceptar las implicaciones entre medio ambiente y desarrollo (Novo 2009, p. 206).

En Tbilisi se adopta un concepto de medio ambiente en el que se incluyen las relaciones de los seres humanos con la naturaleza. Según esta premisa, la EA debería centrarse en la preparación del individuo mediante la comprensión de los principales problemas del mundo contemporáneo, proporcionándole conocimientos técnicos y las cualidades necesarias para desempeñar una función productiva con miras a mejorar la vida y proteger el medio ambiente, prestando la debida atención a los valores éticos (Unesco, 1978, p. 1).

El modelo ético que subyace señala la necesidad de que los individuos se sitúen firmemente en las sociedades en las que viven, tomen conciencia del lugar que ocupa la humanidad en la biosfera, reconozcan las interdependencias recíprocas entre la comunidad de vida y asuman sus responsabilidades. Continúa en la línea marcada por el Seminario de Belgrado de sentar las bases para que ninguna nación crezca y se desarrolle a costa de otras.

Igualmente, se sigue incidiendo en dos cuestiones fundamentales: por un lado, que la EA incluye la educación formal y no formal; $y$, por otro, que los programas deben incluir contenidos teóricos, valores, actitudes, técnicas y comportamientos ecológicos y éticos que permitan reconocer y abordar los problemas del desarrollo sostenible.

Pasada una década, el Congreso Internacional sobre Educación y Formación Ambiental fue organizado en Moscú por la Unesco, el PNUMA y el gobierno de la URSS bajo la denominación Diez años después de Tbilisi. Entre las medidas destaca, por su importancia, el establecimiento de la Comisión Mundial sobre el Medio Ambiente y el Desarrollo, más conocida como Comisión Brundtland (1987). El objetivo de esta Comisión fue estudiar los problemas ambientales de manera global. Para ello se encargaron estudios y se convocaron conferencias públicas en todo el mundo. Tras tres años de trabajo, vio la luz el Informe Brundtland. Una de las aportaciones más relevantes del mismo fue insistir y evidenciar los vínculos existentes entre los 
modelos vigentes de desarrollo y la problemática ambiental. Además, proporcionó la definición más extendida y citada del desarrollo sostenible, si bien parece haber quedado en el olvido la relación ineludible que se establece entre desarrollo sostenible, equidad y participación cuando, por una parte, se indica que es preciso dar prioridad a las necesidades de los más vulnerables $\mathrm{y}$, por otra, se defiende sin posible duda la participación de las personas en la toma de decisiones sobre asuntos socioecológicos que les competen.

Desde posiciones críticas se considera que constituye una contradicción, una manipulación de los «desarrollistas», de los partidarios del crecimiento económico, que pretenden hacer creer en su compatibilidad con la sostenibilidad ecológica, que subordina las exigencias de la economía a las de los ecosistemas naturales y al desarrollo humano generalizado (Naredo, 1996, p. 132). Para Gutiérrez Bastida (2018, p. 41) en el desarrollo conceptual hay una subordinación absoluta del ámbito ecológico y de las comunidades sociales a la economía. Y, para otros autores, con el término desarrollo sostenible se ha producido una cierta colonización del campo que venía ocupando lo que a lo largo de varias décadas hemos denominado educación ambiental (Gutiérrez, Calvo, Benayas, 2006, p. 33). Y que vendría agudizado por la ambigüedad en los discursos -que se inicia a comienzos de la década de los 90, cuando empiezan a aparecer libros y artículos en revistas especializadas que incluyen el concepto en varias versiones: EPS -Educación para la Sostenibilidad-; EFS -Educación para un Futuro Sostenible-; EDS -Educación para el Desarrollo Sostenible-. (Gutiérrez, Calvo, Benayas, 2006, p. 36)

En la Conferencia de Moscú, anteriormente mencionada, la visión del futuro es una de las preocupaciones centrales que contribuye a consolidar la idea del desarrollo sostenible. Los resultados más importantes de esta Conferencia fueron recogidos en la Elementos para una estrategia internacional de acción en materia de educación y formación ambientales para el decenio de 1990. En la primera parte de este documento, se definen los problemas ambientales y las metas de la EA; en la segunda se exponen los principios y las características de la educación y la formación ambiental. Se establecen nueve estrategias de EA que comprenden todos los niveles educativos y que tienen en cuenta la educación formal como no formal.

La década de los 90 supuso la constatación de la gravedad de la crisis socio-ambiental mundial. La Asamblea General de las Naciones Unidas convocó la Conferencia de las Naciones Unidas sobre el Medio Ambiente y el Desarrollo (CNUMAD), en Río de Janeiro en 1992. En esta Conferencia se desarrollaron dos foros paralelos: La Cumbre de la Tierra, a la cual asistieron los Jefes de Estado o representantes gubernamentales de alrededor de 160 países, y el Foro Global, donde la sociedad civil pudo reflexionar sobre los problemas del desarrollo y el medio ambiente, y que reunió a más de 15.000 personas.

Frente a la Declaración de Río o la Agenda21, algunos autores consideran que el documento más relevante emanado del evento es el Tratado de Educación Ambiental para Sociedades Sustentables y Responsabilidad Global, redactado en el marco del Foro Global. En él se considera que las causas primarias de problemas como: el aumento de la pobreza, la degradación humana y ambiental y la violencia, son consecuencias del modelo económico y de civilización imperante. Y que la EA afirma valores y acciones que contribuyen con la transformación humana y social y con la preservación ecológica 
(Foro Global, 1992). Se afirma que la Educación Ambiental no es neutra... es un acto político basado en valores para la transformación social (Foro Global, 1992), afianzando aún más la complejidad de un movimiento que nunca se ha reconocido como educación naturalista.

Cinco años después, en la Conferencia Internacional sobre Ambiente y Sociedad: Educación y Conciencia Pública para la Sustentabilidad, celebrada en Tesalónica en 1997, la Unesco afirmaba la educación ambiental, (...), ha sido además considerada como una educación para la sostenibilidad. Ello permite referirse a ella como una educación para el medio ambiente y la sostenibilidad (Unesco, 1997).

Unesco se decantó por los términos educación para la sostenibilidad o educación para un futuro sostenible, con la oposición de figuras emblemáticas del movimiento ambiental, que no aceptaban esta nueva terminología e insistían en mantener la denominación de educación ambiental como un concepto consolidado e inclusivo de los matices enfatizados en las nuevas denominaciones. Desde entonces, el concepto de educación para el desarrollo sostenible ha ido poco a poco adquiriendo importancia creciente en el escenario internacional.

El Siglo XXI se inicia con la Declaración del Milenio, aprobada por la Asamblea General de las Naciones Unidas el 8 de septiembre del año 2000, en un marco de globalización consolidada y con el avance de los tratados de libre comercio impulsados por la Organización Mundial del Comercio (OMC), entre otras. Además, está presente un proceso de transición de la economía -desde la producción a la especulación- debido a la desregulación de los mercados financieros, (...) la inmaterialidad de los activos financieros, (...) la celeridad de los flujos financieros (Murga-Menoyo, 2013, p. 85) con las nefastas consecuencias que todos conocemos. Quizá por este clima, la Declaración insiste en los valores que han de regir las relaciones internacionales: la libertad, la igualdad, la solidaridad, la tolerancia, el respeto a la naturaleza y la responsabilidad común.

Uno de los frutos de esta Declaración son los Objetivos de Desarrollo del Milenio (ODM) que tratan de consolidar muchos de los compromisos asumidos por separado en las cumbres y conferencias de Naciones Unidas en la década de los 90 (Gutiérrez Bastida, 2018, p. 117), estableciendo para ello plazos, metas e indicadores. Los ODM nacieron con la esperanza de conseguir los avances significativos que no habían logrado instrumentos anteriores, aunque lo cierto es que no han logrado sus metas con eficacia (ONU, 2015a).

En el año 2002, la Asamblea General de las Naciones Unidas aprobó la Resolución 57/254, por la que se proclamaba la Década de las Naciones Unidas de la Educación para el Desarrollo Sostenible (UNSDD, 2005-2014). Esta resolución respondía a un doble desafío: por un lado, las evidencias de la gravedad de los problemas globales que afectaban al planeta y, por otro la constatación de que la lentitud del ritmo en las medidas para afrontar la crisis, muy inferior a su aumento acelerado. Entre los objetivos que se plantearon cabe destacar el fomento de la EDS y su incorporación en las reformas educativas.

Esta resolución supone la prevalencia del término Educación para el Desarrollo Sostenible sobre Educación Ambiental que ya venía tomando forma desde la CNUMAD en 1992. El término se consolida a lo largo del Decenio (UNSDD, 2004-2015), en 
documentos internacionales como la Declaración de Rio+20 (ONU, 2012), el Acuerdo de Mascate (Unesco, 2014), la Declaración de Incheon (Unesco, 2015) o en los propios Objetivos de Desarrollo Sostenible (Longueira, Bautista-Cerro y Rodríguez, 2018).

Frente a las críticas, otras autoras defienden que ofrece un marco amplio, que permite sumar a él cuantos movimientos e iniciativas innovadoras en materia educativa contribuyen a una educación de calidad, en respuesta a las problemáticas sociales y ecológicas, tanto globales como locales, de nuestras sociedades interrelacionadas (Murga-Menoyo, 2015, p. 56).

También en 2012, al cumplirse 20 años de la primera gran Cumbre de la Tierra, se reúne de nuevo en Río un importante número de representantes de todos los países. Sin embargo, el análisis de estadistas y expertos evidencia la serie de promesas incumplidas desde 1992.

En definitiva, desde los primeros intentos en los años setenta, si bien podemos afirmar que la década de los 90 supuso un avance considerable para poner encima de la mesa cuestiones relativas al desarrollo y, por tanto, la necesidad de la EA, la falta de apoyo efectivo de los gobiernos y el agravamiento de las problemáticas en todas las áreas y dimensiones de la sostenibilidad han facilitado que los años finales del siglo $\mathrm{XX}$ y primeros lustros del nuevo siglo estén marcados por los insuficientes avances hacia la sostenibilidad.

Hay que resaltar que, pese al apoyo institucional que ha recibido de forma mayoritaria la educación para el desarrollo sostenible, los movimientos de EAespecialmente en América Latina y el Sur de Europa- se han mantenido firmes no solo en seguir utilizando este concepto y la filosofía de transformación socioecológica que transporta, sino también en las críticas al supuesto crecimiento sostenible que propone el sistema económico dominante y que conduce a una situación de enormes daños ecológicos y sociales, algunos de ellos irreversibles.

En esta línea, las numerosas reuniones y conferencias, así como los documentos emanados de ellas, han servido para hacer avanzar el corpus teórico de la EA y para sustentar los diseños, propuestas y acciones que, sin embargo, en muchas ocasiones, con escaso apoyo oficial, tan solo han llegado a ser realizadas gracias al voluntarismo y la tenacidad de personas comprometidas, convencidas de la necesidad de su trabajo.

\section{La educación ambiental en España}

Si descendemos al nivel nacional, la panorámica es coherente con la anteriormente descrita. La EA comienza a ser una realidad en España hace unos 25 años. En 1983, tuvieron lugar en Sitges (Barcelona) las I Jornadas sobre Educación Ambiental, en las que participaron más de trescientos profesionales. Cuatro años más tarde, como continuación de las anteriores, se celebraron las Jornadas sobre Educación Ambiental en el Estado Español, esta vez en Valsaín. Al año siguiente se llevó a término, en Las Navas del Marqués (Ávila), un Seminario sobre Educación Ambiental en el Sistema Educativo organizado por la Comisión Española de la Unesco y el Ministerio de Educación y Ciencia (MEC). Su objetivo principal era definir las líneas básicas de la Estrategia para introducir la EA en el sistema educativo formal. La posterior Ley de Ordenación General del Sistema Educativo (LOGSE), de 1990, facilitó la inclusión oficial de la EA en la educación formal, como un eje curricular transversal del sistema escolar. 
En España existen distintos elementos (organismos, instituciones) a tener en cuenta en la configuración, evolución y situación de la EA. A nivel institucional, el CENEAM, mantiene abiertas distintas líneas de trabajo sobre EA (investigación, formación y difusión). Es un referente indispensable a la hora de trabajar sobre estos temas. Por otro lado, las Asociaciones de Educadores y Educadoras Ambientales también han mantenido una actividad digna de mención.

La elaboración de la Estrategia Nacional de Educación Ambiental, contenida en el Libro Blanco de la Educación Ambiental, supuso un acontecimiento de gran relevancia en el panorama de la EA. En 1993, el ICONA realizó un primer acercamiento a esta temática mediante una investigación recogida en el libro Bases para una Estrategia Española de Educación Ambiental, coordinado por María Novo. Seis años más tarde, en 1999, vería la luz el Libro Blanco de la Educación Ambiental en España, con la voluntad clara de impulsar este movimiento educativo. El documento pretende ser un marco de referencia y establece las líneas de actuación para los diferentes actores y organismos implicados.

Entre los aspectos que considera relevantes para la calidad de la educación, el Libro Blanco desataca el mayor peso de los factores sociales en los programas educativos; la necesidad de hacer hincapié en el cambio de valores y comportamientos; la extensión de la EA en todos los ámbitos; la integración de la educación ambiental en la política ambiental y el fomento de la participación ciudadana.

El Libro Blanco aborda un análisis sectorial de los diferentes ámbitos de actuación de la EA. Se plantea la necesidad de acciones coordinadas entre los diferentes agentes y sectores sociales.

Recomienda impulsar el reconocimiento profesional de los educadores ambientales como técnicos cualificados para la planificación, ejecución y/o evaluación de programas de educación ambiental (Comisión Temática de Educación Ambiental, 1999 , p. 108). Señalando que se trata de una cuestión de gran interés para los educadores ambientales que, hasta el momento, ha encontrado una respuesta minoritaria tanto a nivel público como privado; y que la crisis económica de los últimos años ha supuesto una quiebra importante de la que aun los equipamientos y programas de educación ambiental no se han recuperado.

Por otro lado, defiende la necesidad de ampliar y consolidar una oferta de formación universitaria de postgrado que permita acceder a una especialización en gestión y EA complementada con tareas investigadoras de calidad. (Comisión Temática de Educación Ambiental, 1999, p. 96).

Por lo que respecta a la educación formal, lo cierto es que el reconocimiento y presencia de la EA no ha encontrado ni el hueco ni el apoyo necesario. La LOGSE introdujo los temas ambientales de manera transversal, un mandato que lamentablemente no llegó a consolidarse y que fue desapareciendo en las posteriores normativas. La Ley Orgánica de Educación (LOE) o la actual Ley Orgánica para la Mejora de la Calidad Educativa (LOMCE) han ido expulsando la EA del currículo, totalmente centrado en la formación de competencias y en los logros de aprendizaje-. Si bien los objetivos de la EA tienen plena cabida tanto en unas como en otros, al no estar explícitamente demandados por normativa quedan en la práctica a la voluntad del profesorado. 
Nos dice José Manuel Gutiérrez que la educación ambiental no vino para quedarse. En algún momento -una vez asimilados culturalmente sus objetivos- pasará a ser simplemente 'educación' sin necesidad del adjetivo. A este respecto existe un movimiento en el ámbito de la educación superior, que defiende la necesidad de formar a los estudiantes en las competencias y valores de la sostenibilidad, para que sean capaces de afrontar los desafíos ambientales con la formación necesaria.

En España, el Grupo de Trabajo Sostenibilización Curricular de la Conferencia de Rectores de las Universidades Españolas (CRUE) lleva años trabajando para propiciar la inclusión de la sostenibilidad en el currículo universitario. La sostenibilización curricular, supone no solo educar sobre sostenibilidad sino también educar para la sostenibilidad. Y, en ese sentido, es necesario facilitar la adquisición por los estudiantes del conjunto complejo e integrado de conocimientos, destrezas, habilidades, actitudes y valores que las personas ponen en juego en los distintos contextos (sociales, educativos, laborales, familiares) para resolver situaciones relacionadas con las problemáticas ambientales, así como de operar y transformar la realidad con criterios de sostenibilidad (Geli, Junyent y Sánchez, 2004, p. 229); es decir, de las competencias en sostenibilidad.

La universidad es cada vez más consciente de su importante papel para abordar la crisis ambiental y son numerosas las iniciativas que se han ido desarrollando en este sentido (Bautista-Cerro y Díaz, 2017).

No podemos cerrar este epígrafe sin mencionar los grandes referentes de la EA en las universidades españolas. El primer programa específico de Postgrado, el Máster Internacional en Educación Ambiental, inició su andadura en 1990, de la mano de María Novo, profesora titular de la UNED. Y continuará su andadura el marco de la Cátedra Unesco de Educación Ambiental y Desarrollo Sostenible de la UNED en el momento en que sea crea esta Cátedra, año 1996. Durante décadas ha sido el referente en formación no solo a nivel estatal sino también fuera de España, especialmente en América Latina, manteniéndose vigente durante 25 años.

En el curso 2000-2001 apareció el Programa Interuniversitario de Educación Ambiental que, con la participación de nueve universidades, mantuvo la formación de doctores en EA hasta que la introducción del Plan Bolonia modificó sustancialmente la organización de los estudios universitarios. En la actualidad, la oferta de estudios oficiales de postgrado se mantiene con el Máster Interuniversitario en Educación Ambiental, coordinado por la Universidad de Cádiz, y en el que participan distintas universidades andaluzas.

\section{¿Dónde estamos ahora? La Agenda 2030 una nueva herramienta}

La Agenda 2030, que se encuentra desde el 2015 sobre la mesa, es un instrumento que está recibiendo una buena acogida en gobiernos e instituciones públicas y privadas. Esta Agenda es un documento aprobado por la Asamblea General de Naciones Unidas cuyo objetivo es liberar a la humanidad de la tiranía de la pobreza y las privaciones, así como sanar y proteger nuestro planeta (ONU, 2015b). La Agenda contiene 17 objetivos (Objetivos de Desarrollo Sostenible, ODS) que pretenden guiar nuestro mundo hacia la sostenibilidad durante el periodo 2016-2030. Estos 17 ODS se concretan en 169 metas orientadas a lograr el desarrollo mundial y una cooperación en la que todos salgan ganando (ONU, 2015b, p. 6). Tanto los objetivos 
como las metas se encuentran interconectados y el avance debe ser, sin duda, coordinado ante la magnitud de los problemas que se intenta contrarrestar.

Estamos resueltos a construir un futuro mejor para todos (...) Tal vez seamos la primera generación que consiga poner fin a la pobreza, y quizás seamos también la última que todavía tenga posibilidades de salvar el planeta. Si logramos nuestros objetivos, el mundo será un lugar mejor en 2030 (ONU, 2015b, p. 14).

Como señala Murga-Menoyo (2018), sin negar sus limitaciones, la Agenda 2030 y sus objetivos presentan elementos positivos respecto a sus inmediatos predecesores, los Objetivos de Desarrollo del Milenio (ODM) (2001-2015). Especialmente relevante es el cambio copernicano del punto de mira. Los ODS superan el antiguo enfoque de cooperación al desarrollo que impregnaba los ODM al plantear los objetivos y metas como resolución de problemáticas que interpelan directamente a todos y cada uno de los países del globo, con independencia de su avance tecnológico y económico. Adicionalmente, ponen en el centro del discurso el concepto de sostenibilidad; y lo hacen desde una perspectiva glocal, en decir, local y global a un tiempo. Ningún país será reconocido cumplidor de la Agenda 2030 si no minimiza, hasta eliminarlo, su impacto ecológico y social sobre territorios ajenos (...), la aprobación de los ODS supone un impulso hacia la universalización, en este aspecto, de la justicia ambiental (MurgaMenoyo, 2018, p. 40).

La EA podrá, pues, verse beneficiada con el avance de la Agenda 2030. Por un lado, porque ésta última evidencia los problemas ambientales, su carácter sistémico y la necesidad de actuación desde distintos frentes, cada uno de los cuales se convierte en objetivo de desarrollo sostenible, entre cuyas metas siempre encontramos alguna que menciona la necesidad instrumental de la educación para su logro. Es, pues, la educación, un eje transversal a todos los ODS; les afecta de manera individual y colectiva.

Por otra parte, la educación tiene asignado en la Agenda un objetivo específico el no 4 Garantizar una educación inclusiva, equitativa, y de calidad y promover oportunidades de aprendizaje durante toda la vida para todos (ONU, 2015, p. 16). Con una meta, la número 7, que hace referencia implícita a la EA: De aquí a 2030, asegurar que todos los estudiantes adquieran los conocimientos teóricos y prácticos necesarios para promover el desarrollo sostenible (ONU, 2015, p. 20).

Ahora bien, es preciso aceptar que la Agenda y los ODS también son objeto de críticas por no implicar cambios más radicales y profundos. Se argumenta que no suponen ninguna crítica ni cuestionamiento del modelo de crecimiento y reparto de la riqueza dominante, limitándose a introducir cambios que, en definitiva, lo consolidan sin contrarrestar las causas reales de los problemas. No cabe negar que es posible encontrar contradicciones en su discurso, algunas de las cuales ya estaban presentes en numerosos documentos internacionales anteriores. Nos referimos, por ejemplo, al planteamiento del crecimiento sostenible (sic), uno de los conceptos más debatidos y discutidos desde posiciones no solo ambientales, sino también económicas alternativas. La mención explícita, en el ODS número 8, al Trabajo decente y crecimiento económico valida y alienta el modelo actual, causante de la crisis en la que estamos inmersos (ONU, 2015a, p. 22). 
Sin embargo, y pese a las críticas, los ODS están teniendo una importante repercusión en la reflexión sobre los estilos de vida y la calidad de vida, a nivel discursivo y como generadores de debate. Gobiernos e instituciones públicas y privadas están planteando cambios, aunque tímidos, en la dirección que requieren estos objetivos. Se trata, por tanto, de una herramienta útil para que la EA obtenga una mayor difusión y vea ampliando su campo de acción.

\section{A modo de conclusión}

La historia de la EA es la de un movimiento con un largo e intenso recorrido y está marcada, por un lado, por la gran cantidad de eventos internacionales y, por otro, por las aportaciones individuales, constantes y tenaces de los profesionales que le han dedicado y dedican su trabajo. La mayoría de los planteamientos teóricos que han ido apareciendo, cumbre tras cumbre, siguen teniendo vigencia hoy en día y, aunque gran parte de ellos se plasman en el trabajo de los educadores ambientales, el desarrollo de programas de formación o las políticas públicas muy puntuales, lo cierto es que a nivel institucional los avances no se han desarrollado con la profundidad que sería necesaria.

Tras décadas de reuniones y conferencias, solo algunas de las cuales hemos incluido en este breve repaso, la EA con sus limitaciones y sin que muchos lo hayan querido ver así, nunca ha dejado de posicionarse como una práctica pedagógica y social contrahegemónica -y, tal vez por ello, del todo necesaria- orientada a un mejor desarrollo de la Humanidad Cartea y Gómez (2006, p. 115). Las dificultades del avance de la EA provienen, en parte de ese enfoque, pero también están relacionadas con el aumento de la complejidad de nuestras sociedades y los problemas socioecológicos que experimentamos, lo que convierte a la EA en un ámbito interdisciplinar en el que confluyen todas las ciencias.

Igualmente es necesario reseñar las tensiones internas en un campo siempre en construcción y pendiente de una consolidación que no parece llegar. La EA experimenta tensiones internas relacionadas con su definición, como hemos visto con la controversia terminológica, y que llenan de matices los discursos incluso de los educadores y educadoras más reconocidos. También externas, al ser desvirtuada muchas veces por las exigencias de programas y proyectos centrados exclusivamente en lo ecológico. En estos casos puede convertirse en una EA estética y poco crítica, que es demandada casi como producto de ocio y tiempo libre y no favorece el crecimiento de actuaciones que responda verdaderamente a la esencia de la EA: dedicar tiempo a fomentar la comprensión de las causas de los problemas ambientales, a reflexionar, a trabajar el espíritu crítico, (...) a capacitar para la acción..., de tal forma que nos hemos quedado en el activismo ambiental con poca o nula reflexión-acción (Gutiérrez Bastida, 2018, p. 91).

Más allá del necesario examen crítico no podemos dejar de congratularnos por la pervivencia de un movimiento que nos habla de contención en un mundo que persigue la opulencia, el despilfarro y la inequidad; de redistribución en el siglo de la concentración y el acaparamiento de bienes; de justicia social cuando asistimos al drama de las migraciones forzadas por la destrucción de muchos hábitats. Pervive en colegios, institutos, universidades, granjas escuelas, oficinas de parques nacionales, 
movimientos vecinales, asociaciones, redes de docentes. Y también está presente en Congresos y Simposios, en las conversaciones y en los discursos.

El ritmo de extinción de las especies, el cambio climático y sus consecuencias en todos los planos de la vida, han acallado los planteamientos negacionistas y han legitimado las tesis que desde la EA se vienen difundiendo desde hace décadas: la necesidad de un cambio en las relaciones del ser humano con el medio natural y con sus congéneres. Este cambio es necesario y ha de ser abordarlo no solo mediante la educación. Pero, probablemente, la educación ambiental sea un elemento sin el cual resulta imposible el camino hacia la sostenibilidad.

\section{Referencias bibliográficas}

Bautista-Cerro, M. J. y Díaz, M. J. (2017). La sostenibilidad en los grados universitarios: presencia y coherencia. Teoría de la educación. Revista Interuniversitaria, 29(1), 161-187.

Carson, R. (1962). Primavera silenciosa. Barcelona: Grijalbo.

Comisión Mundial sobre el Medio Ambiente y el Desarrollo (1987). Nuestro futuro común. Madrid: Alianza.

Comisión temática de Educación Ambiental (1999). Libro Blanco de la Educación Ambiental en España. Madrid: MEC.

Duarte, C. M. (coord.) (2006). Cambio Global. Impacto de la actividad humana sobre el sistema Tierra. Madrid: CSIC.

Foro Global (1992). Tratado de Educación Ambiental para Sociedades Sustentables y Responsabilidad Global.

Geli, A. M., Junyent, M. y Sánchez, S. (eds.) (2004). Diagnóstico de la Ambientalización Curricular de los Estudios Superiores. Girona, Servei de Publicacions Universitat de Girona/Red ACES.

Gimeno Sacristán, J. (2001). Educar y convivir en la cultura global. Las exigencias de la ciudadanía. Madrid: Morata.

Gutiérrez Bastida, J. M. (2011). Sus tenere. Sostenibilidad vs. Mercado y Tecnología. Madrid: Bubok.

Gutiérrez Bastida, J. M. (2018). Educatio ambientalis: Invitación a la educación ecosocial en el Antropoceno. Madrid: Bubok.

Gutiérrez, J., Calvo, S. y Benayas, J. (2006). Educación para el desarrollo sostenible: evaluación de retos y oportunidades del decenio 2005-2014. Revista Iberoamericana de Educación. 40, 25-69.

Harari, Y. N. (2015). Sapiens. De animales a dioses. Barcelona: Debate.

Hardin, G (1968). The Tragedy of the Commons. Science. 162(3859), 1243-1248.

Laval, C. y Dardot, P. (2015). Común. Barcelona: Gedisa.

Longueira, S., Bautista-Cerro, M. J. y Rodríguez Hernández, J. A. (2018). La educación para el desarrollo sostenible: Sin tiempo para educar en el futuro, educando para la emergencia del presente. En C. Barroso (Coord.). Educación en la 
sociedad del conocimiento y el desarrollo sostenible, (pp. 19-54). La Laguna: Universidad de La Laguna.

Meadows, D. H., Meadows, D. L. y Randers J. L. (1972). Los límites del crecimiento (Informe al Club de Roma). México D. F.: Fondo De Cultura Económica.

Meira Cartea, P. A. y Caride Gómez, J. A. (2006). La geometría de la educación para el desarrollo sostenible, o la imposibilidad de una nueva cultura ambiental. Revista Iberoamericana de Educación, 41. 103-116.

Murga-Menoyo, M. A. (2013). Desarrollo Sostenible. Problemáticas, agentes y estrategias. Madrid: McGraw Hill/ UNED.

Murga-Menoyo, M. A. (2015). Competencias para el desarrollo sostenible: las capacidades, actitudes y valores meta de la educación en el marco de la Agenda global post-2015. Foro de Educación, 13(19), 55-83.

Murga-Menoyo, M. A. (2018). La formación de la ciudadanía en el Marco de la Agenda 2030 y la justicia ambiental. Revista Internacional de Educación para la Justicia Global. 07 (01), 37-52.

Naredo, J. M. (1996). Sobre el origen, el uso y el contenido del término sostenible. En La construcción de la ciudad sostenible, Madrid: Ministerio de Obras Públicas, Transportes y Medio Ambiente.

Novo, M. (1993). Bases para una estrategia española de Educación Ambiental. Madrid: ICONA.

Novo, M. (2006). El desarrollo sostenible: su dimensión ambiental y educativa. Madrid: Pearson/Unesco.

Novo, M. (2009). La educación ambiental: una genuina educación para el desarrollo sostenible, Revista de Educación, número extraordinario, 195-217.

ONU (2015a). Objetivos de Desarrollo del Milenio. Informe 2015. N. Y.: Naciones Unidas.

ONU (2015b). Transformar nuestro mundo: La agenda 2030 para el desarrollo sostenible. A/RES/70/1 de 21 de octubre de 2015.

Sartori, G. (1998). Homo videns. La sociedad teledirigida. Barcelona: Taurus.

Unesco (1978). Conferencia Intergubernamental sobre Educación Ambiental. (URSS) Octubre. Informe final. DOc. Ed/MD. 49. Paris: UNESCO.

Unesco (1997). Declaración de Tesalónica. Draf. Doc. EPD-97/CONF.401/CLD.2. París: Unesco.

Unesco (2014). Declaración final de la Reunión Mundial sobre la EPT de 2014 El Acuerdo de Mascate. ED-14/EFA/ME/3.

Unesco (2015). Declaración de Icheon. Foro Mundial sobre la Educación. ED2016/WS/28.

Unesco/PNUMA (1987). Elementos para una estrategia internacional de acción en materia de educación y formación ambientales para el decenio de 1990. París: Unesco/PNUMA.

Vitousek, P. M., Mooney, H. A., Lubchenco, J. y Melillo, J. M. (1997). Human Domination of Earths Ecosystems, Science, 277(25), 494-499. 